\title{
Extracts of Phyllostachys pubescens Leaves Represses Human Steroid 5-Alpha Reductase Type 2 Promoter Activity in BHP-1 Cells and Ameliorates Testosterone-Induced Benign Prostatic Hyperplasia in Rat Model
}

\author{
Kwang Hoon Song ${ }^{1, *(\mathbb{D})}$, Chang-Seob Seo ${ }^{1} \mathbb{D}$, Won-Kyung Yang ${ }^{2} \mathbb{D}$, Hyun-O Gu $^{2} \mathbb{D}$, Ki-Joong Kim ${ }^{2}$ \\ and Seung-Hyung Kim $^{2}$ (D) \\ 1 Herbal Medicine Research Division, Korea Institute of Oriental Medicine, Yuseong-daero 1672, \\ Yuseong-gu, Daejeon 34054, Korea; csseo0914@kiom.re.kr \\ 2 Institute of Traditional Medicine and Bioscience, Daejeon University, Daejeon 34520, Korea; \\ ywks1220@dju.kr (W.-K.Y.); rich1169@naver.com (H.-O.G.); cowking1122@naver.com (K.-J.K.); \\ sksh518@dju.kr (S.-H.K.) \\ * Correspondence: ksong@kiom.re.kr; Tel.: +82-42-868-9390
}

\section{check for} updates

Citation: Song, K.H.; Seo, C.-S.; Yang, W.-K.; Gu, H.-O; Kim, K.-J.; Kim, S.-H. Extracts of Phyllostachys pubescens Leaves Represses Human Steroid 5-Alpha Reductase Type 2 Promoter Activity in BHP-1 Cells and Ameliorates Testosterone-Induced Benign Prostatic Hyperplasia in Rat Model. Nutrients 2021, 13, 884. https://doi.org/10.3390/nu13030884

Academic Editor: Lynnette Ferguson

Received: 28 December 2020

Accepted: 5 March 2021

Published: 9 March 2021

Publisher's Note: MDPI stays neutral with regard to jurisdictional claims in published maps and institutional affiliations.

Copyright: (c) 2021 by the authors. Licensee MDPI, Basel, Switzerland. This article is an open access article distributed under the terms and conditions of the Creative Commons Attribution (CC BY) license (https:// creativecommons.org/licenses/by/ $4.0 /)$.

\begin{abstract}
Benign prostatic hyperplasia (BPH) is the most common symptomatic abnormality of the human prostate characterized by uncontrolled proliferation of the prostate gland. In this study, we investigated the effect of bamboo, Phyllostachys pubescens, leaves extract (PPE) on human $5 \alpha$-reductase type 2 (SRD5A2) gene promoter activity in human prostate cell lines and the protective effect of PPE on a testosterone-induced BPH rat model. PPE repressed human SRD5A2 promoter activity and its mRNA expression. The rats treated with PPE for 4 weeks showed a significantly attenuated prostate weight compared to vehicle control. PPE-treated rats also showed reduced serum dihydrotestosterone, testosterone, prostate-specific antigen, and SRD5A2 levels by testosterone injection. Quantitative realtime polymerase chain reaction showed that PPE treatment significantly decreased mRNA expression of SRD5A2, androgen receptor (AR), proliferating cell nuclear antigen (PCNA), and fibroblast growth factor 2 compared with the vehicle-treated, testosterone-injected rats in the prostate. Furthermore, PPE treatment showed reduced AR, PCNA, and tumor necrosis factor alpha expression in the prostate via immunohistofluorescence staining. In conclusion, oral administration of PPE prevented and inhibited the development and progression of enlarged prostate lesions in testosterone-induced animal models through various anti-proliferative and anti-inflammatory pharmacological effects and induced suppression of SRD5A2 gene expression.
\end{abstract}

Keywords: Phyllostachys pubescens; benign prostatic hyperplasia; $5 \alpha$-reductase type 2 ; dihydrotestosterone

\section{Introduction}

Benign prostatic hyperplasia $(\mathrm{BPH})$ is the most common symptomatic abnormality of the human prostate that affects older men [1]. Many men over the age of 40 have histologically identifiable $\mathrm{BPH}$, and the prevalence increases with age, leading to impaired urination [2]. The main treatment for an enlarged prostate is prostatectomy through laparotomy or urethral resection, but it is often associated with a number of complications, including urinary tract infections, sexual dysfunction, or bleeding [3,4].

While studies to develop a clear and detailed pathogenesis of $\mathrm{BPH}$ are ongoing, previous studies have shown that male hormones such as androgens, testosterone, and specifically dihydrotestosterone (DHT) are linked to the onset of BPH [5]. The typical role of $5 \alpha$-reductase is to metabolize testosterone to $5 \alpha$-dihydrotestosterone, the most potent androgen [6]. $5 \alpha$-reductase promotes the $5 \alpha$-position reduction of 4 -ene-3-keto steroids such as testosterone, 4 -androstenedione, and progesterone [7]. DHT performs 
important physiological functions such as differentiation of the male external genitalia and secondary growth during puberty, especially in men, but it has been demonstrated to be involved in androgen-dependent diseases $[8,9]$. There are diseases such as prostate hypertrophy, prostate cancer, and skin diseases such as male hair loss, seborrheic dermatitis, and acne $[1,10]$. To prevent or treat these diseases, inhibiting DHT generation in target cells is thought to be effective, and various $5 \alpha$-reductase enzyme inhibitors such as finasteride and dutasteride have been developed and are used in the treatment of BPH [6]. However, these drugs are known to cause adverse effects such as erectile dysfunction, loss of libido, myopathy, and chest pain [11,12].

Recent research suggests that herbs used in oriental medicine can effectively treat $\mathrm{BPH}$ or inhibit the occurrence of $\mathrm{BPH}$ [13-15]. These herbal extracts contain alkaloids, flavonoids, isoflavones, saponins, and other phenolics. With increasing interest in the use of functional foods and dietary supplements for health care, saw palmetto extract is widely used as a functional food to suppress prostate enlargement [16]. However, a product extracted from saw palmetto only had a placebo effect in improving the symptoms of an enlarged prostate and urinary tract and it did not improve the symptoms of an enlarged prostate $[16,17]$. Therefore, the development of functional food materials with safety and functionality is required.

Bamboo is known to be effective against obesity, and it also relaxes the mind and helps relieve stress, insomnia, and forgetfulness [18,19]. It is also known for its anti-inflammatory, antioxidant, anti-osteoporosis, and anti-wrinkle effects, and it is helpful for hypertension, arteriosclerosis, and activating innate immunity $[20,21]$. Bamboo leaves are a medicinal herb containing various active ingredients such as flavonoids, phenolic acids, and coumarin lactone [22]. However, the efficacy of bamboo leaves for BPH has not yet been studied. Therefore, in this study, we evaluated the effect of bamboo, Phyllostachys pubescens, leaves extract (PPE) on SRD5A2 gene expression in human prostate cell lines and a testosteroneinduced $\mathrm{BPH}$ rat model.

\section{Materials and Methods}

\subsection{Luciferase Reporter Constructs}

Human SRD5A2 promoter fragments from - 1642 to $-1 \mathrm{bp}$ were constructed in the pGL3 luciferase vector. A human genomic fragment spanning from -1642 to -1 bp of SRD5A2 was amplified by PCR using the following oligonucleotides: forward 5'- CCCGGGCTCGAGATC TGAAACGGCTATGATGGCT- ${ }^{\prime}$ and reverse ${ }^{\prime}$ ' - CCGGAATGCCAAGCTTCGCGCCGTGTT CCTCGCC-3'; the fragment was then digested with BgIII and HindIII and was infused to the PGL3 basic vector between the BgIII and HindIII sites, and the construction of the amplified DNA sequences was verified by DNA sequencing.

\subsection{Preparation of Phyllostachys pubescens Extract (PPE)}

Dried leaves of P. pubescens were provided from Nova K-Med Co. Ltd. (Daejeon, Korea). The origin of the sample was confirmed by Dr. Goya Choi, the Herbal Medicine Resources Research Center, Korea Institute of Oriental Medicine (KIOM; Naju, Korea). The voucher specimen (2020PPL) has been deposited at the Herbal Medicine Research Division, KIOM. To prepare PPE, dried P. pubescens leaves were extracted with $80 \%$ ethanol for $3 \mathrm{~h}$, the extract was filtered, and the organic solvent was removed. Then, it was freeze-dried to obtain a powder sample.

\subsection{Cell Culture}

Human prostate epithelial cells, BPH-1 cells, were obtained from Creative Bioarray (Shirley, NY, USA). The cells were maintained in an RPMI1640 (Life technologies, Carlsbad, CA, USA), supplemented with $1 \%$ penicillin/streptomycin (Gibco, Grand Island, NY, USA) and $20 \%$ heat-inactivated fetal bovine serum (Gibco by Life Technologies, USA) at $37{ }^{\circ} \mathrm{C}$ in an atmosphere containing $5 \% \mathrm{CO}_{2}$. 


\subsection{Transient Transfection and Luciferase Reporter Assay}

For the luciferase reporter assay, BPH-1 cells were plated in 48 -well plates $24 \mathrm{~h}$ before transfection; then, the cells were transfected with the luciferase reporter using the TranslTLT1 transfection reagent (Mirus Bio, Madison, WI, USA) according to the manufacturer's instructions. For reporter quantitation, cell lysates were assayed using the Luciferase Assay System (Promega, Madison, WI, USA) and a TriStar LB941 Luminometer (Berthold technologies, Bad Wildbad, Germany) according to the manufacturer's instructions.

\subsection{RNA Isolation and Quantitative Real-Time PCR (Q-PCR)}

Total RNA was isolated using the RNeasy Mini Kit (Qiagen, Hilden, Germany) according to the manufacturer's instructions. To detect relative mRNA expression, reversetranscription and Q-PCR were performed with TaqMan probes (Life Technologies, USA) as described previously [23] or with SYBR Premix (Takara, Kusatsu, Japan). Relative amounts of cDNA were calculated by using the relative quantification $(\Delta \Delta \mathrm{Ct})$ method. Experiments were repeated at least three times with different cell preparations. The sequences of synthesized PCR primer sets (Bioneer Co. Ltd., Seoul, Korea) for rat 5a-reductase type 2 were $5^{\prime}$ GACCACAGGCGAGATGCAGA-3' and $5^{\prime}$-TGTGTTTCCCGTAACTGGCG-3' ; for rat proliferating cell nuclear antigen (PCNA), they were $5^{\prime}$-CAATTTCTAGCAACGCCTAAGAT- $3^{\prime}$ and $5^{\prime}$-AAGAGGAAGCTGTGTCCATAGAG-3'; for rat androgen receptor (AR), they were $5^{\prime}$-CAAAGGGTTGGAAGGTGAGA-3' and $5^{\prime}$-GAGCGAGCGGAAAGTTGTAG-3'; and for rat fibroblast growth factor (FGF2), they were $5^{\prime}$-GAACCGGTACCTGGCTATGA- $3^{\prime}$ and $5^{\prime}$-CCGTTTTGGATCCGAGTTTA- $3^{\prime}$. The relative abundance of each transcript was normalized to that of ubiquitin $\mathrm{C}$ or GAPDH.

\subsection{Cytotoxicity Assay}

BPH-1 cells were seeded into 48-well plates $\left(2 \times 10^{4}\right.$ cells/well $)$ and incubated for $24 \mathrm{~h}$. The following day, a medium containing PPE (at concentrations ranging from $10-300 \mu \mathrm{g} / \mathrm{mL}$ ) was added. Twenty-four hours later, the cell proliferation rate was determined using the CellTiter $96^{\circledR}$ AQueous One Solution Cell Proliferation Assay (Promega, Wisconsin, WI, USA), according to the manufacturer's protocol as previously described [23].

\subsection{Animals, BPH Induction, and PPE Administration}

Male Sprague-Dawley rats (6 weeks old) were purchased from Dae Han Bio Link Co., Ltd. (Chungcheongbuk-do, Korea). After a 1-week acclimatization period, the animals were housed and kept under a controlled temperature $\left(22 \pm 2{ }^{\circ} \mathrm{C}\right)$ and humidity $(55 \pm 15 \%)$, on a 12-h light/dark cycle. All animal procedures were performed in accordance with the guidelines for the Care and Use of Laboratory Animals developed by Institute of Laboratory Animal Resources of the National Research Council and were approved by the Institutional Animal Care and Use Committee of Daejeon University (DJUARB2020-017) in Daejeon, Korea.

To prevent the effects of endogenous testosterone, rats in all groups except the control $(\mathrm{CON})$ rats group underwent bilateral orchiectomy 2 days before testosterone propionate (Sigma-Aldrich, St Louis, MO, USA) injection. For BPH studies, castrated rats were randomly divided into 5 groups of 8 animals, with equal distribution of body weight. Prostate hyperplasia was induced by subcutaneous injection of testosterone propionate $(5 \mathrm{mg} / \mathrm{kg}$ ) daily for 4 weeks. During the 4 weeks of the prostatic hyperplasia induction period, three of the groups were treated with three oral doses $(50,100$, and $200 \mathrm{mg} / \mathrm{kg}$ ) of PPE, while the positive drug control group received finasteride $(100 \mathrm{mg} / \mathrm{kg})$.

Rats were fasted overnight prior to being sacrificed, with blood samples collected via cardiac puncture of anesthetized rats. The serum was separated from blood cells via centrifugation and was stored at $-80^{\circ} \mathrm{C}$ until analysis. The prostate was immediately excised, weighed, and stored at $-80^{\circ} \mathrm{C}$. Other tissues were also excised and rinsed with phosphate buffered saline (PBS) to remove blood from the tissues, flash-frozen in liquid nitrogen, and then stored at $-80^{\circ} \mathrm{C}$ until analysis. 


\subsection{Histological Analysis}

For histopathological examination, the prostate was fixed in $10 \%$ formalin and prepared into paraffin blocks. Sections from the blocks with a thickness of $4 \mu \mathrm{m}$ were stained with hematoxylin and eosin (H\&E) and observed with an optical microscope.

\subsection{Serum Analysis}

Serum was separated from the blood via centrifugation at $1500 \times g$ for $10 \mathrm{~min}$. The content of testosterone, DHT, prostate-specific antigen (PSA), and SRD5A2 in the serum of each group was analyzed by ELISA kit (Mybiosource, San Diego, CA, USA) according to the manufacturer's protocols. Serum GPT (glutamate pyruvate transaminase, also known as alanine aminotransferase, ALT) and GOT (glutamate oxaloacetate transaminase, also known as aspartate transaminase, AST) were measured using an automatic clinical chemistry analyzer (XL-200, Erba Diagnostics Mannheim, Germany) using $100 \mu \mathrm{L}$ of heart blood serum.

\subsection{Immunohistofluorescence Staining}

To perform immunohistofluorescence staining, the extracted prostate tissue was cut into a $20-\mu \mathrm{m}$ thick section at $-20{ }^{\circ} \mathrm{C}$ using a frozen sectioner and then attached to the slide. To fix the tissue, $4 \%$ paraformaldehyde (Sigma-Aldrich, St Louis, MO, USA) and $4 \%$ sucrose (Sigma-Aldrich, St Louis, MO, USA) were added and fixed at room temperature for $45 \mathrm{~min}$. $10 \mathrm{mM}$ Glycine (Sigma-Aldrich, St Louis, MO, USA) was added to PBST (a solution containing $0.1 \%$ Triton X-100 in PBS) and washed three times for 5 min. Thereafter, $0.5 \% \mathrm{NP}-40$ was added to PBS and reacted for $30 \mathrm{~min}$ at room temperature. After washing for 5 min with PBST, a blocking buffer with $5 \%$ goat serum (Biowest, Riverside, MO, USA), $5 \%$ horse serum (Biowest, Riverside, MO, USA), and 3\% bovine serum albumin (Sigma-Aldrich, St Louis, MO, USA) was prepared in PBS-T solution. The blocking reaction was carried out overnight at $4{ }^{\circ} \mathrm{C}$ with the solution. The primary antibody was diluted in blocking buffer, reacted at room temperature for $4 \mathrm{~h}$, and washed three times with PBST for $10 \mathrm{~min}$ each. After, the secondary antibody was also diluted in a blocking buffer, reacted for $2 \mathrm{~h}$ at room temperature under conditions in which light was blocked, and washed once for 10 min with PBST. Nuclear staining was incubated with Hoechst 33258. Images were acquired using a fluorescence microscope (Nikon, Japan) and ACT-1 software. Primary antibodies used for staining were AR (SCBT, Dallas, TX, USA, 1:400), PCNA (SCBT, Dallas, TX, USA, 1:400), and TNF $\alpha$ (SCBT, Dallas, TX, USA, 1:400). Secondary antibodies used were fluorescein goat antimouse IgG antibody $(\mathrm{H}+\mathrm{L})$ (Invitrogen, Carlsbad, CA, USA; 1:400) and rhodamine goat antirabbit antibody (Invitrogen, Carlsbad, CA, USA; 1:400).

\subsection{Chemicals and Reagents}

Five reference standard compounds were purchased from commercial suppliers: chlorogenic acid (99.6\%) from Acros Organics (Pittsburgh, PA, USA), isoorientin (98.5\%) from Shanghai Sunny Biotech (Shanghai, China), orientin (99.1\%) and isovitexin $(99.3 \%)$ from Biopurify Phytochemicals (Chengdu, China), and 4-hydroxycinnamic acid (99.2\%) from Wako Chemicals (Osaka, Japan). Solvents (methanol, acetonitrile, and water) used were HPLC-grade and purchased from JT Baker (Phillipsburg, NJ, USA). Formic acid and dimethyl sulfoxide used were ACS reagent grade and purchased from Merck KGaA (Darmstadt, Germany).

\subsection{HPLC Analysis of Five Marker Components in P. pubescens Leaves}

The HPLC analysis of two phenylpropanoids (chlorogenic acid and 4-hydroxycinnamic acid) and three flavonoids (isoorientin, orientin, and isovitexin) for quality evaluation of $P$. pubescens leaves was performed in a Shimadzu Prominence LC-20A series (Kyoto, Japan) equipped with two pumps (LC-20AT), an online degasser (DGU-20A3), a column oven (CTO-20A), automatic sample injector (SIL-20A), and a photodiode array detector (SPD-M20A). Five marker analytes were separated by using Gemini C18 (Phenomenex, 
$4.6 \times 250 \mathrm{~mm}, 5 \mu \mathrm{m}$, Torrance, CA, USA) maintained at $40{ }^{\circ} \mathrm{C}$. The mobile phases were eluted by gradient condition with distilled water (solvent $\mathrm{A}$ ) and acetonitrile (solvent $\mathrm{B}$ ); both contained $0.1 \%(v / v)$ formic acid. The gradient elution condition of the mobile phase was as follows: $5 \%-60 \%$ B for $0-20 \mathrm{~min}, 60 \%$ B for $25-30 \mathrm{~min}$, and $60 \%-5 \%$ B for $30-35 \mathrm{~min}$. The flow rate and injection volume of standard and sample solutions were $1.0 \mathrm{~mL} / \mathrm{min}$ and $10 \mu \mathrm{L}$, respectively. All data were acquired and processed by LabSolution software (version 5.53, SP3, Kyoto, Japan).

\subsection{Statistical Analysis}

Statistical analysis was performed using GraphPad Prism 7 (GraphPad Software, San Diego, CA, USA). Normality of the distributions was evaluated using Shapiro-Wilk's test. As the samples followed normal distribution, between-group differences were evaluated using a one-way analysis of variance (ANOVA), followed by Dunnett's test to identify significant differences between the BPH and CON groups. Significant $p$-values are reported as follows: * and \#, $p<0.05 ;{ }^{* *}$ and \#\#, $p<0.01$; and ${ }^{* * *}$ and \#\#\#, $p<0.001$.

\section{Results}

\subsection{PPE Suppresses Human 5 $\alpha$-Reductase Type 2 Promoter Activity in the Human BPH} Epithelial Cell Line BPH-1

We constructed a human $5 \alpha$-reductase type 2 (SRD5A2) gene promoter containing reporter luciferase to screen for substances that can be controlled at the stage of the SRD5A2 gene expression, not to search for substances that inhibit the enzyme activity of $5 \alpha$-reductase. Since natural extracts have long been recognized as a potential source of treatment, cell-based reporter screening tests have been used to select extracts that inhibit human SRD5A2 promoter activity in BPH-1 cells.

To identify these extracts, BPH-1 cells were plated on the 48-well plate, delivered with the human SRD5A2 promoter reporter, and treated with 200 natural extracts. After $24 \mathrm{~h}$ of incubation, luciferase activity was measured. We focused on herbal extracts that inhibited the SRD5A2 promoter activity by less than 50\% compared to the control. Initial hits were retested according to dose (Figure 1A). We identified that PPE, an herbal extract, inhibits more than $50 \%$ of the human SRD5A2 promoter luciferase activity from this screening.
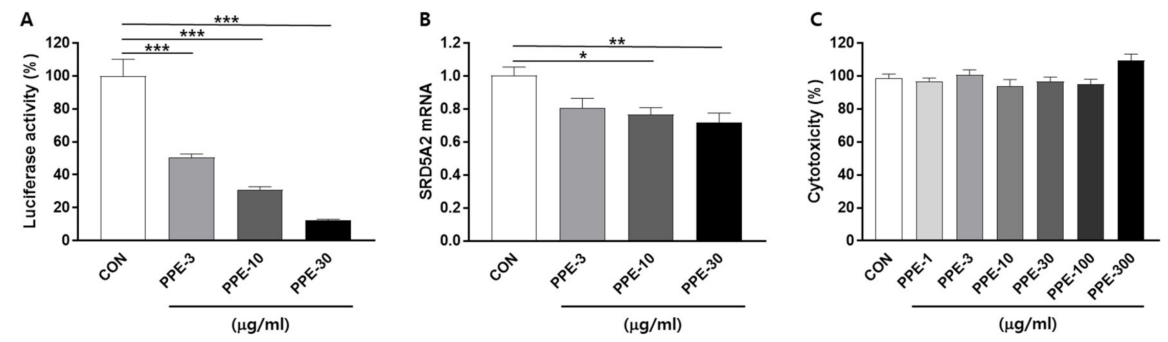

Figure 1. Phyllostachys pubescens leaves extract (PPE) suppressed human SRD5A2 promoter activity and mRNA expression. (A) Concentration-response effects of PPE on human SRD5A2 promoter activity. BPH-1 cells were transfected with the luciferase reporter plasmid vectors comprising the human SRD5A2 promoter sequence. Cells were treated with the indicated concentrations of PPE for $24 \mathrm{~h}$ and luciferase activity was measured by a luminometer. (B) mRNA levels of SRD5A2 were measured by Q-PCR. (C) Cytotoxicity effects of PPE. All data are shown as mean \pm S.E.M. of three independent experiments with triplicate wells. ${ }^{*} p<0.05,{ }^{* *} p<0.01$, and ${ }^{* *} p<0.001$, compared with CON.

To further confirm whether PPE represses the human SRD5A2 gene at a transcriptional level, SRD5A2 mRNA levels in BPH-1 cells were measured by quantitative real-time PCR (Q-PCR). Seeding BPH-1 cells in 6-well plates and treatment with PPE $(3,10$ and $30 \mu \mathrm{g} / \mathrm{mL})$ for $24 \mathrm{~h}$ resulted in reduced SRD5A2 mRNA expression in a dose-dependent manner (Figure 1B). 
The cytotoxicity of PPE was verified using an MTS assay, which showed that treatment with PPE at concentrations up to $300 \mu \mathrm{g} / \mathrm{mL}$ for $24 \mathrm{~h}$ did not significantly affect BPH-1 cell viability (Figure 1C).

\subsection{Effects of PPE on Prostate Weights}

To further investigate whether PPE has a protective effect on testosterone-induced $\mathrm{BPH}$ in vivo, PPE was administrated in three different doses (50, 100, and $200 \mathrm{mg} / \mathrm{kg} /$ day) for 4 weeks. Consistent with previous reports, rats injected with testosterone for 4 weeks induced prostatomegaly (Figure 2A,B). Prostate weight in testosterone-injected rats was about 2-fold greater than that of the control group rats, but treatment with PPE (100 and $200 \mathrm{mg} / \mathrm{kg} /$ day) significantly attenuated prostate weight compared to the testosteroneinjected group (BPH) alone. Positive controls, finasteride $(1 \mathrm{mg} / \mathrm{kg} /$ day $)$, also showed an inhibitory effect on prostate weight compare to the BPH group. We also analyzed the prostatic index which was calculated as the ratio of prostate weight to body weight (Figure 2C). PPE (100 and $200 \mathrm{mg} / \mathrm{kg} /$ day) and finasteride ( $1 \mathrm{mg} / \mathrm{kg} /$ day) also significantly inhibited the testosterone-induced prostatic index.

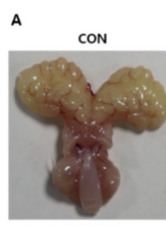

PPE-100
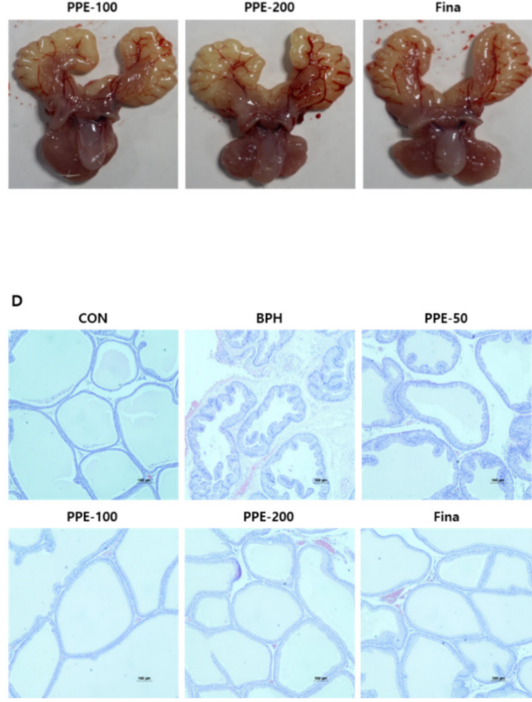

B
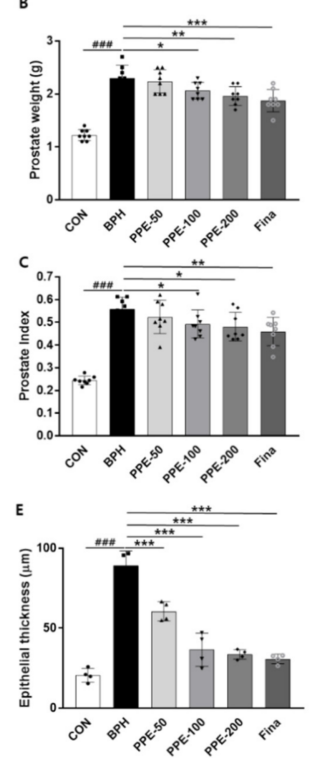

Figure 2. PPE suppressed testosterone-induced prostate enlargement. Castrated rats were given an oral dose of PPE $(50,100$, and $200 \mathrm{mg} / \mathrm{kg})$ or finasteride $(1 \mathrm{mg} / \mathrm{kg})$ with $5 \mathrm{mg} / \mathrm{kg}$ of testosterone injected daily. Rats injected with the vehicle were used as the negative control (CON) group. (A) Representative prostate images were shown from each experimental group. (B) Prostate weight was measured and analyzed. All data are mean $\pm \mathrm{SD}(n=8$ per group). (C) The prostatic index was calculated as the ratio of prostate weight to body weight. (D) Representative prostatic tissue slide-stained by hematoxylin and eosin (H\&E) was shown (magnification $\times 100)$. (E) The epithelial thickness of prostate was measured and the graph is expressed as the mean \pm SD of 4 rats per experimental group.

We further confirmed the inhibitory effects of PPE on prostatomegaly by histological analysis (Figure 2D,E). In the prostate tissue of the control group (CON), one layer of low columnar epithelial cells constitutes the luminal endocrine cells, and the cavity is filled with a light eosinophilic substance, but the prostate epithelial tissue thickness was increased in the BPH group that caused prostate enlargement with testosterone. On the other hand, the PPE-treated group and finasteride group showed reduced epithelial tissue thickness 
compared to the BPH group. These data indicated that PPE was effective in reducing prostate enlargement in testosterone-induced rats.

\subsection{Effects of PPE on Serum Prostate Parameters Levels}

The finding that PPE supplementation ameliorated prostatomegaly in BPH rats prompted us to evaluate the change in serum prostate parameters (Figure 3). The serum prostate parameters, DHT, testosterone, PSA, and SRD5A2 levels, were significantly increased in BPH group rats compared to control group rats. However, PPE treatment remarkably inhibited these serum prostate parameter levels compared to the BPH group in a dose-dependent manner. These results confirmed that PPE treatment restored the normal form of the prostate in BPH by reducing the increase in serum DHT, testosterone, PSA, and SRD5A2 levels by testosterone injection.
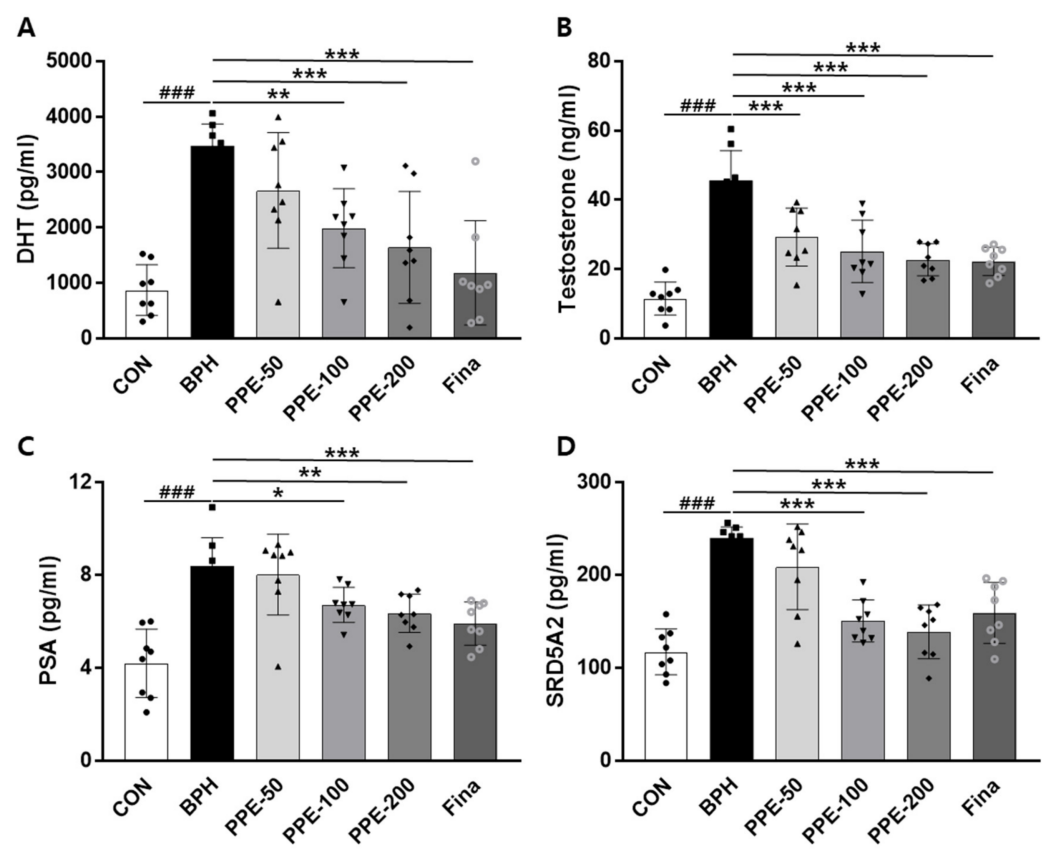

Figure 3. Effect of PPE on DHT, testosterone, PSA, and SRD5A2 levels in serum. The concentration of (A) DHT, (B) testosterone, (C) PSA, and (D) SRD5A2 were analyzed using an ELISA kit. Data are presented as mean $\pm \mathrm{SD}(n=8)$. \#\#\# $p<0.001$ compared with control group, and values with different letters indicate significant differences, ${ }^{*} p<0.05,{ }^{* *} p<0.01$, and ${ }^{* * *} p<0.001$, compared with BPH group.

\subsection{Analysis of SRD5A2, AR, PCNA, and FGF2 $m R N A$ Levels in Prostate}

We examined the effects of PPE on SRD5A2, AR, PCNA, and FGF2 mRNA expression in prostate tissue via Q-PCR (Figure 4). Injection of testosterone (BPH) markedly increased the mRNA expression of SRD5A2, AR, PCNA, and FGF2 compared to the CON group. PPE treatment significantly decreased mRNA expression of SRD5A2 (PPE, 100 and $200 \mathrm{mg} / \mathrm{kg} /$ day), AR (PPE, $200 \mathrm{mg} / \mathrm{kg} /$ day), PCNA (PPE, 100 and $200 \mathrm{mg} / \mathrm{kg} /$ day), and FGF2 (PPE, $200 \mathrm{mg} / \mathrm{kg} /$ day). Significant change in the mRNA levels of SRD5A2, AR, PCNA, and FGF2 was also observed in the finasteride-treated group. Taken together, PPE was effective to prevent testosterone-induced BPH in rats not only by inhibiting SRD5A2 expression but also by inhibiting AR-dependent prostate proliferation. 
A

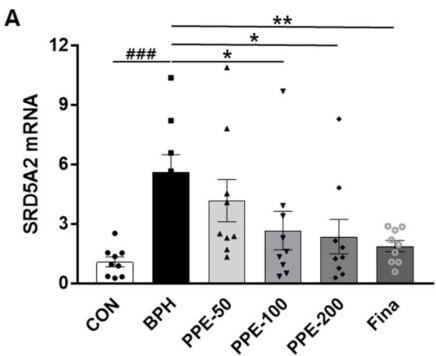

C

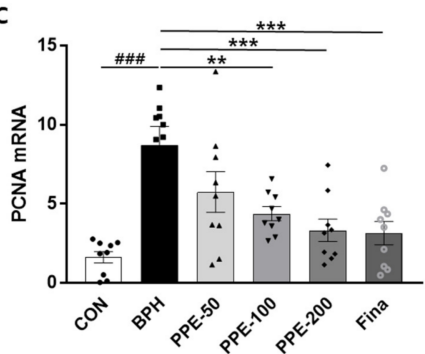

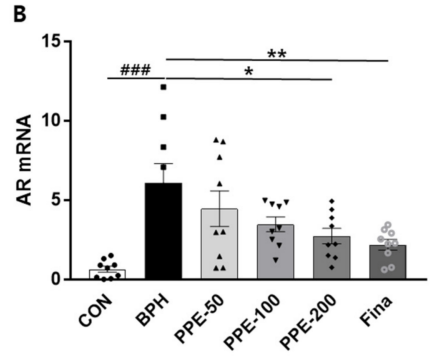

D

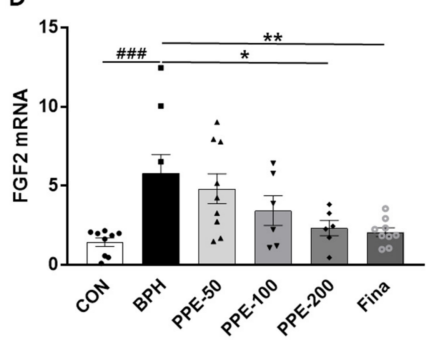

Figure 4. Effect of PPE on mRNA levels of SRD5A2, AR, PCNA, and FGF2 in the prostate. The expression of prostatic genes (A) SRD5A2, (B) AR, (C) PCNA, and (D) FGF2 were addressed by real-time quantitative PCR. Data are presented as mean $\pm \operatorname{SD}(n=8)$. \#\#\# $p<0.001$ compared with control group, and values with different letters indicate significant differences, ${ }^{*} p<0.05,{ }^{* *} p<0.01$, and ${ }^{* * *} p<0.001$, compared with the BPH group.

\subsection{Effect of PPE on AR, PCNA, and TNFa Expression in Prostate}

In addition, we examined the effects of PPE on androgen-related gene (AR, PCNA, and $\mathrm{TNF} \alpha$ ) expression in prostate tissue via IHF. As shown in Figure 5, dramatically increased $\mathrm{AR}, \mathrm{PCNA}$, and TNF $\alpha$-positive cells in the epithelium prostate by testosterone injection $(\mathrm{BPH})$ were observed. However, the testosterone-mediated upregulation of AR, PCNA, and TNF $\alpha$ expression were reduced significantly in the PPE- and finasteride-treated groups.
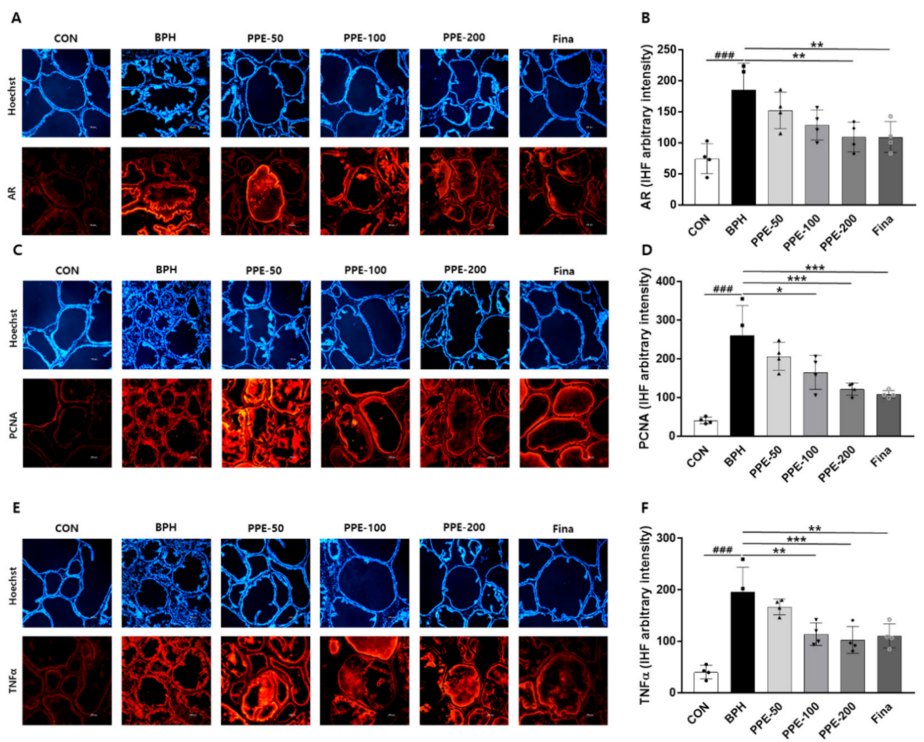

Figure 5. Effect of PPE on AR, PCNA, and TNF $\alpha$ expression in prostate. The expression of $(\mathbf{A}, \mathbf{B}) \mathrm{AR}$, (C,D) PCNA, and (E,F) TNF $\alpha$ were analyzed by IHF. Representative immunofluorescence images were shown and colabeled with Hoechst 33,258 (blue). Magnification 10×; scale bar, $100 \mu \mathrm{m}$. The densities of proteins were calculated using ACT-1 software. Data represent arbitrary intensity relative to $\mathrm{CON}$ (control) as the mean $\pm \mathrm{SD}$ of 4 rats per experimental group. \#\#\# $p<0.001$ compared with control group, and values with different letters indicate significant differences, ${ }^{*} p<0.05,{ }^{* *} p<0.01$, and ${ }^{* * *} p<0.001$, compared with the BPH group. 


\subsection{Hepatotoxicity of PPE in Rat BPH Model}

To address the effect of PPE on liver damage, serum enzyme activities of GTP and GOT were evaluated (Figure 6). The serum GTP and GOT levels were not significantly changed in $\mathrm{BPH}$-group rats compared to the control-group rats. By confirming the decrease in GPT and GOT in the PPE treatment group, we speculated the possibility that PPE could be helpful in reducing hepatotoxicity.

A

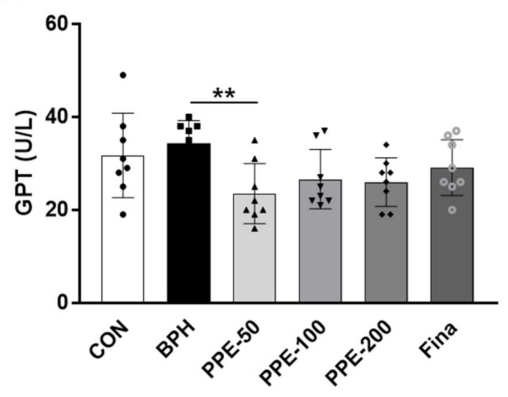

B

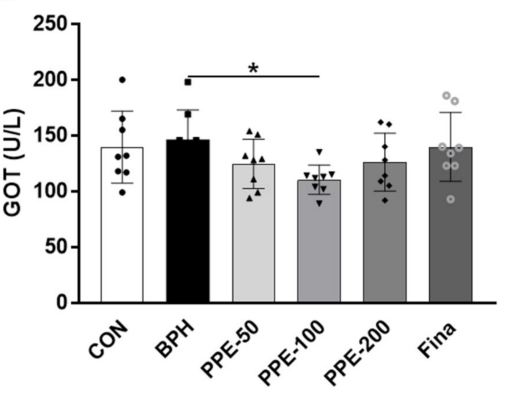

Figure 6. Effect of PPE on serum GTP and GOT levels. (A) Serum GPT and (B) GOT levels at 4 weeks after PPE treatment are shown. Data are presented as mean $\pm \operatorname{SD}(n=8)$. Values with different letters indicate significant differences, ${ }^{*} p<0.05$ and ${ }^{* *} p<0.01$, compared with the BPH group.

\subsection{Quantification of Five Marker Components in P. pubescens Leaves}

In the established HPLC assay, the peak of each compound was identified by comparing it with the retention time and UV spectrum of each reference standard compound. All components were separated within $20 \mathrm{~min}$ and a representative HPLC chromatogram was shown in Figure 7. Five marker components (chlorogenic acid, isoorientin, orientin, isovitexin, and 4-hydroxycinnamic acid) were simultaneously analyzed in P. pubescens leaves samples by established the HPLC-analysis assay. Quantification of these analytes was conducted at $315 \mathrm{~nm}$ for 4-hydrocinnamic acid, $325 \mathrm{~nm}$ for chlorogenic acid, $335 \mathrm{~nm}$ for isovitexin, and $350 \mathrm{~nm}$ for isoorientin and orientin. The amounts of the five marker components in the lyophilized $80 \%$ ethanol extract of $P$. pubescens leaves were $1.71-11.63 \mathrm{mg} / \mathrm{g}$ (Table 1).

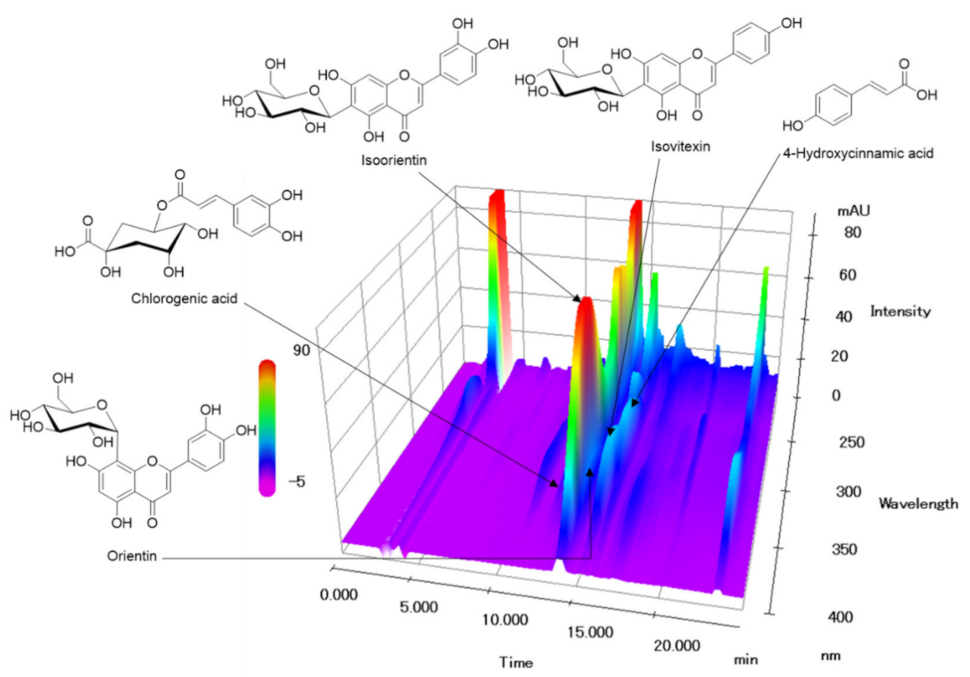

Figure 7. Three-dimensional HPLC chromatogram of P. pubescens leaves. Five marker components, chlorogenic acid, isoorientin, orientin, isovitexin, and 4-hydroxycinnamic acid, were detected at 12.24, $13.92,14.34,15.30$, and $15.66 \mathrm{~min}$, respectively. 
Table 1. Amounts of the five marker analytes in P. pubescens leaves samples $(n=3)$.

\begin{tabular}{|c|c|c|c|c|c|c|}
\hline \multirow{3}{*}{ Analyte } & \multicolumn{6}{|c|}{ Batch no. } \\
\hline & \multicolumn{2}{|c|}{1} & \multicolumn{2}{|c|}{2} & \multicolumn{2}{|c|}{3} \\
\hline & $\begin{array}{c}\text { Mean }(\mathrm{mg} / \mathrm{g}) \pm \\
\operatorname{SD}^{\mathrm{a}}\left(\times 10^{-1}\right)\end{array}$ & $\operatorname{RSD}^{b}(\%)$ & $\begin{array}{c}\text { Mean }(\mathrm{mg} / \mathrm{g}) \pm \\
\operatorname{SD}\left(\times 10^{-1}\right)\end{array}$ & RSD (\%) & $\begin{array}{c}\text { Mean }(\mathrm{mg} / \mathrm{g}) \pm \\
\operatorname{SD}\left(\times 10^{-1}\right)\end{array}$ & RSD (\%) \\
\hline Chlorogenic acid & $1.71 \pm 0.08$ & 0.44 & $1.85 \pm 0.16$ & 0.84 & $1.78 \pm 0.17$ & 0.95 \\
\hline Isoorientin & $10.94 \pm 0.18$ & 0.16 & $11.63 \pm 0.23$ & 0.20 & $11.29 \pm 0.30$ & 0.27 \\
\hline Orientin & $3.06 \pm 0.03$ & 0.09 & $3.25 \pm 0.09$ & 0.29 & $3.15 \pm 0.02$ & 0.07 \\
\hline Isovitexin & $4.36 \pm 0.15$ & 0.34 & $4.65 \pm 0.06$ & 0.12 & $4.51 \pm 0.09$ & 0.20 \\
\hline 4-Hydroxycinnamic acid & $2.13 \pm 0.04$ & 0.17 & $2.27 \pm 0.07$ & 0.30 & $2.20 \pm 0.10$ & 0.44 \\
\hline
\end{tabular}

a SD means standard deviation. ${ }^{b}$ RSD means relative standard deviation and presented as SD/Mean $\times 100$.

\section{Discussion}

In this research, we investigated the effect of PPE on human SRD5A2 promoter activity in human BPH-1 cells and on testosterone-induced BPH in rats. We found that PPE inhibits human SRD5A2 promoter activity and attenuates testosterone-induced prostatic hypertrophy and pathological changes in prostate tissue by reducing the expression of SRD5A2 as well as serum DHT levels, and the results were comparable to those in the finasteridetreated group used as a positive control. Moreover, testosterone-induced expression of AR, PCNA, and TNF $\alpha$ in the prostate was inhibited by PPE treatment. Therefore, these results suggest that PPE has an effective anti-proliferative effect as a therapeutic agent for prostatic hyperplasia.

$\mathrm{BPH}$ occurs due to the overgrowth of prostate epithelial cells and stromal cells as men age, which is known to be caused by an imbalance between cell proliferation and apoptosis. DHT is known to be the most important male hormone for prostatic hypertrophy, and SRD5A2 regulates the process of DHT formation from testosterone. For this reason, SRD5A2 has been studied as an important target in the development of many prostate hypertrophy inhibitors. Finasteride, a well-known BPH treatment drug so far, also targets SRD5A2 to reduce blood DHT and reduce prostate size [24]. However, taking finasteride for a long period of time has been known to cause unwanted adverse effects and has warranted the development of a new drug. Therefore, many studies have been conducted to obtain clinical evidence for alternative treatment of BPH. Botanical remedies are drawing attention as part of an effort to replace synthetic drugs and minimize side effects.

Steroid hormones such as testosterone and DHT regulate various cellular processes such as cell growth, proliferation, and differentiation [25]. It is also known to play an important role in the development of BPH by AR, and modulating AR signaling could be a major treatment method for BPH [25]. The testosterone-induced BPH rats had increased serum DHT production and $5 \alpha$-reductase levels and increased expression of AR in prostate tissue, and its activation may imply interaction with endogenous androgens. However, PPE significantly reduced the expression of AR and showed a significant inhibitory effect on the expression of $5 \alpha$-reductase consistent with the target-based cellular study that showed PPE inhibited SRD5A2 promoter activity. PCNA is a nuclear protein induced during the G1/S phase transition, acts as a marker for cell proliferation, and plays an important role in physiological conditions such as BPH $[26,27]$. In this study, the cell proliferation process was compared and analyzed to find out the mechanism of the pathogenesis of BPH using PCNA and FGF2. The Q-PCR and IHF results of PCNA showed that PPE significantly downregulated the expression of PCNA-positive cells in prostate tissue. These observations suggest the anti-proliferative effect of PPE on cell-cycle progressions such as the inhibition of RB/E2F, which is generally associated with the regulation of transcription of DNA synthesis and G1/S regulatory gene activity. Testosterone-induced FGF2 mRNA levels in the prostate were also markedly suppressed by PPE administration, similar to the action of finasteride. Since the regulation of FGF2 is dependent on DHT, inhibition of FGF2 expression by PPE is assumed to be the effect of reduced DHT through inhibition 
of SRD5A2 expression. These results suggest that PPE may be an effective prostatic hyperplasia treatment.

The area where prostate enlargement occurs is known as the area where chronic inflammation occurs frequently [28]. This chronic inflammatory environment has emerged as one of the important causes of prostatic hyperplasia, and inflammation in prostatic hyperplasia is known to be associated with patient symptoms, the progression of prostate enlargement, and elevated PSA, a prostate indicator protein. Since serum PSA levels are frequently elevated in prostate diseases such as BPH and prostate cancer, they have therefore been used as clinical indicators of disease prognosis [29]. In our study, testosterone-induced BPH rats also showed a significant increase in serum PSA levels. However, oral administration of PPE markedly reduced the serum PSA levels compared to the BPH group, suggesting the prevention of prostatic hypertrophy progression. Since AR occupied by a ligand such as DHT regulates prostate proliferation, while simultaneously upregulating PSA expression, it is thought that the reduction of DHT through PPE-mediated SDR5A2 inhibition consequently suppressed the expression of PSA. A significant reduction in TNF $\alpha$ in the prostate of the PPE treatment group suggests that anti-inflammatory agents are involved in the mechanism of PPE in BPH treatment, but more investigation of other cytokines will be needed to further reinforce our conclusions. Moreover, although further studies of potential chronic toxicity and genotoxicity are required, PPE treatment resulted in no significant increase in serum hepatic parameters, GPT and GOT, which indicates that PPE does not cause hepatotoxicity in rats up to $200 \mathrm{mg} / \mathrm{kg}$ of PPE.

P. pubescens has been commonly used as a traditional herb in Korea, Japan, and China [30-32], and we identified that PPE contained five phytochemicals: chlorogenic acid, isoorientin, orientin, isovitexin, and 4-hydroxycinnamic acid. These ingredients are known to have pharmacological effects including anticancer [33], antioxidant [34], anxiolytic activity [35], and anti-inflammatory [36] effects. Although we have not clearly identified the compounds responsible for the efficacy of PPE, multiple target activities of PPE on BPH suggest that it may involve synergistic or additional effects of several active ingredients in PPE.

\section{Conclusions}

In conclusion, oral administration of PPE prevented and inhibited the development and progression of enlarged prostate lesions in testosterone-induced animal models through various anti-proliferative and anti-inflammatory pharmacological effects as well as through suppression of SRD5A2 gene expression. Although it should be confirmed through human application studies, our findings suggest that PPE may be a potential herbal medicine such as a dietary supplement for BPH therapy that effectively slows the progression of $\mathrm{BPH}$.

Author Contributions: K.H.S. conceived, designed the experiments, and prepared the manuscript; K.H.S., C.-S.S., W.-K.Y., H.-O.G., K.-J.K. and S.-H.K. performed the experiments and analyzed the data; All authors have read and agreed to the published version of the manuscript.

Funding: This research was funded by the Korea Institute of Oriental Medicine, grant number KSN20134262.

Institutional Review Board Statement: All animal procedures were performed in accordance with the guidelines for the Care and Use of Laboratory Animals developed by Institute of Laboratory Animal Re-sources of the National Research Council and were approved by the Institutional Animal Care and Use Committee of Daejeon University (DJUARB2020-017) in Daejeon, Korea.

Informed Consent Statement: Not applicable.

Data Availability Statement: The data presented in this study are available from the corresponding author on reasonable request.

Conflicts of Interest: The authors declare no conflict of interest. 


\section{References}

1. Roehrborn, C.G. Benign prostatic hyperplasia: An overview. Rev. Urol. 2005, 7 (Suppl. 9), S3-S14. [PubMed]

2. Lee, C.L.; Kuo, H.C. Pathophysiology of benign prostate enlargement and lower urinary tract symptoms: Current concepts. Ci Ji Yi Xue Za Zhi Tzu Chi Med. J. 2017, 29, 79-83. [CrossRef]

3. Xing, N.; Guo, Y.; Yang, F.; Tian, L.; Zhang, J.; Yan, Y.; Kang, N.; Xin, Z.; Niu, Y. Laparoscopic simple prostatectomy with prostatic urethra preservation for benign prostatic hyperplasia. Transl. Androl. Urol. 2012, 1, 9-13. [CrossRef] [PubMed]

4. Carbone, D.J.; Hodges, S. Medical therapy for benign prostatic hyperplasia: Sexual dysfunction and impact on quality of life. Int. J. Impot. Res. 2003, 15, 299-306. [CrossRef] [PubMed]

5. Nicholson, T.M.; Ricke, W.A. Androgens and estrogens in benign prostatic hyperplasia: Past, present and future. Differ. Res. Biol. Divers. 2011, 82, 184-199. [CrossRef] [PubMed]

6. Azzouni, F.; Godoy, A.; Li, Y.; Mohler, J. The 5 alpha-reductase isozyme family: A review of basic biology and their role in human diseases. Adv. Urol. 2012, 2012, 530121. [CrossRef]

7. Rižner, T.L.; Penning, T.M. Role of aldo-keto reductase family 1 (AKR1) enzymes in human steroid metabolism. Steroids 2014, 79, 49-63. [CrossRef]

8. Patrão, M.T.C.C.; Silva, E.J.R.; Avellar, M.C.W. Androgens and the male reproductive tract: An overview of classical roles and current perspectives. Arq. Bras. Endocrinol. Metabol. 2009, 53, 934-945. [CrossRef]

9. Handelsman, D.J.; Hirschberg, A.L.; Bermon, S. Circulating Testosterone as the Hormonal Basis of Sex Differences in Athletic Performance. Endocr. Rev. 2018, 39, 803-829. [CrossRef]

10. Lai, J.J.; Chang, P.; Lai, K.P.; Chen, L.; Chang, C. The role of androgen and androgen receptor in skin-related disorders. Arch. Dermatol. Res. 2012, 304, 499-510. [CrossRef]

11. Hirshburg, J.M.; Kelsey, P.A.; Therrien, C.A.; Gavino, A.C.; Reichenberg, J.S. Adverse Effects and Safety of 5-alpha Reductase Inhibitors (Finasteride, Dutasteride): A Systematic Review. J. Clin. Aesthetic Dermatol. 2016, 9, 56-62.

12. Kaplan, S.A.; Chung, D.E.; Lee, R.K.; Scofield, S.; Te, A.E. A 5-year retrospective analysis of $5 \alpha$-reductase inhibitors in men with benign prostatic hyperplasia: Finasteride has comparable urinary symptom efficacy and prostate volume reduction, but less sexual side effects and breast complications than dutasteride. Int. J. Clin. Pract. 2012, 66, 1052-1055. [CrossRef]

13. Kim, J.H.; Park, K.M.; Lee, J.A. Herbal medicine for benign prostatic hyperplasia: A protocol for a systematic review of controlled trials. Medicine 2019, 98, e14023. [CrossRef]

14. Shin, S.J.; Lee, K.H.; Chung, K.S.; Cheon, S.Y.; An, H.J. The traditional Korean herbal medicine Ga-Gam-Nai-Go-Hyan suppresses testosterone-induced benign prostatic hyperplasia by regulating inflammatory responses and apoptosis. Exp. Ther. Med. 2017, 13, 1025-1031. [CrossRef] [PubMed]

15. Shin, I.S.; Lee, M.Y.; Ha, H.K.; Seo, C.S.; Shin, H.-K. Inhibitory effect of Yukmijihwang-tang, a traditional herbal formula against testosterone-induced benign prostatic hyperplasia in rats. Bmc Complement. Altern. Med. 2012, 12, 48. [CrossRef] [PubMed]

16. Fagelman, E.; Lowe, F.C. Saw Palmetto Berry as a Treatment for BPH. Rev. Urol. 2001, 3, 134-138. [PubMed]

17. Barry, M.J.; Meleth, S.; Lee, J.Y.; Kreder, K.J.; Avins, A.L.; Nickel, J.C.; Roehrborn, C.G.; Crawford, E.D.; Foster, H.E., Jr.; Kaplan, S.A.; et al. Effect of increasing doses of saw palmetto extract on lower urinary tract symptoms: A randomized trial. JAMA 2011, 306, 1344-1351. [CrossRef]

18. Kim, D.S.; Kim, S.H.; Cha, J. Antiobesity Effects of the Combined Plant Extracts Varying the Combination Ratio of Phyllostachys pubescens Leaf Extract and Scutellaria baicalensis Root Extract. Evid. Based Complement. Altern. Med. eCAM 2016, 2016, 9735276. [CrossRef]

19. Son, E.; Kim, S.H.; Yang, W.K.; Kim, D.S.; Cha, J. Antiplatelet mechanism of an herbal mixture prepared from the extracts of Phyllostachys pubescens leaves and Prunus mume fruits. Bmc Complement. Altern. Med. 2017, 17, 541. [CrossRef]

20. Choi, S.; Park, M.S.; Lee, Y.R.; Lee, Y.C.; Kim, T.W.; Do, S.G.; Kim, D.S.; Jeon, B.H. A standardized bamboo leaf extract inhibits monocyte adhesion to endothelial cells by modulating vascular cell adhesion protein-1. Nutr. Res. Pract. 2013, 7, 9-14. [CrossRef]

21. Fang, J.; Gao, S.; Islam, R.; Teramoto, Y.; Maeda, H. Extracts of Phellinus linteus, Bamboo (Sasa senanensis) Leaf and Chaga Mushroom (Inonotus obliquus) Exhibit Antitumor Activity through Activating Innate Immunity. Nutrients 2020, 12, 2279. [CrossRef] [PubMed]

22. Lu, B.; Wu, X.; Shi, J.; Dong, Y.; Zhang, Y. Toxicology and safety of antioxidant of bamboo leaves. Part 2: Developmental toxicity test in rats with antioxidant of bamboo leaves. Food Chem. Toxicol. Int. J. Publ. Br. Ind. Biol. Res. Assoc. 2006, 44, 1739-1743. [CrossRef]

23. Yin, J.; Seo, C.S.; Hwang, I.H.; Lee, M.W.; Song, K.H. Anti-Obesity Activities of Chikusetsusaponin IVa and Dolichos lablab L. Seeds. Nutrients 2018, 10, 1221. [CrossRef] [PubMed]

24. Nickel, J.C. Comparison of clinical trials with finasteride and dutasteride. Rev. Urol. 2004, 6 (Suppl. 9), S31-S39. [PubMed]

25. Sciarra, A.; Mariotti, G.; Salciccia, S.; Autran Gomez, A.; Monti, S.; Toscano, V.; Di Silverio, F. Prostate growth and inflammation. J. Steroid Biochem. Mol. Biol. 2008, 108, 254-260. [CrossRef]

26. Strzalka, W.; Ziemienowicz, A. Proliferating cell nuclear antigen (PCNA): A key factor in DNA replication and cell cycle regulation. Ann. Bot. 2011, 107, 1127-1140. [CrossRef]

27. Zhong, W.; Peng, J.; He, H.; Wu, D.; Han, Z.; Bi, X.; Dai, Q. Ki-67 and PCNA expression in prostate cancer and benign prostatic hyperplasia. Clin. Investig. Med. Med. Clin. Exp. 2008, 31, E8-E15. [CrossRef] [PubMed]

28. Chughtai, B.; Lee, R.; Te, A.; Kaplan, S. Role of inflammation in benign prostatic hyperplasia. Rev. Urol. $2011,13,147-150$. 
29. Manji, M. Prostate-specific antigen (PSA): An overview. Ann. Saudi Med. 2002, 22, 1-3. [CrossRef]

30. Koide, C.L.; Collier, A.C.; Berry, M.J.; Panee, J. The effect of bamboo extract on hepatic biotransforming enzymes-findings from an obese-diabetic mouse model. J. Ethnopharmacol. 2011, 133, 37-45. [CrossRef]

31. Fu, S.G.; Yoon, Y.; Bazemore, R. Aroma-active components in fermented bamboo shoots. J. Agric. Food Chem. 2002, 50, 549-554. [CrossRef]

32. Yong-Chun, J.; Lin, Y.; Yuan, K. A novel high-performance liquid chromatography fingerprint approach to discriminate Phyllostachys pubescens from China. Pharmacogn. Mag. 2012, 8, 42-48. [CrossRef] [PubMed]

33. Yang, H.; Said, A.M.; Huang, H.; Papa, A.P.D.; Jin, G.; Wu, S.; Ma, N.; Lan, L.; Shangguan, F.; Zhang, Q. Chlorogenic acid depresses cellular bioenergetics to suppress pancreatic carcinoma through modulating c-Myc-TFR1 axis. Phytother. Res. PTR 2020. [CrossRef] [PubMed]

34. Ziqubu, K.; Dludla, P.V.; Joubert, E.; Muller, C.J.F.; Louw, J.; Tiano, L.; Nkambule, B.B.; Kappo, A.P.; Mazibuko-Mbeje, S.E. Isoorientin: A dietary flavone with the potential to ameliorate diverse metabolic complications. Pharmacol. Res. 2020, $158,104867$. [CrossRef]

35. Wang, X.; Chen, Y.; Wang, Q.; Sun, L.; Li, G.; Zhang, C.; Huang, J.; Chen, L.; Zhai, H. Support for Natural Small-Molecule Phenols as Anxiolytics. Molecules 2017, 22, 2138. [CrossRef]

36. Liu, B.; Huang, B.; Hu, G.; He, D.; Li, Y.; Ran, X.; Du, J.; Fu, S.; Liu, D. Isovitexin-Mediated Regulation of Microglial Polarization in Lipopolysaccharide-Induced Neuroinflammation via Activation of the CaMKK $\beta /$ AMPK-PGC- $1 \alpha$ Signaling Axis. Front. Immunol. 2019, 10, 2650. [CrossRef] [PubMed] 